\title{
A!
}

This is an electronic reprint of the original article.

This reprint may differ from the original in pagination and typographic detail.

Kong, Beibei; Yla-Oijala, Pasi; Sihvola, Ari

\section{Surface Integral Equation Method for Generalized Soft-and-Hard Boundary Condition}

Published in:

IEEE Transactions on Antennas and Propagation

DOI:

10.1109/TAP.2019.2963587

Published: 01/05/2020

Document Version

Peer reviewed version

Please cite the original version:

Kong, B., Yla-Oijala, P., \& Sihvola, A. (2020). Surface Integral Equation Method for Generalized Soft-and-Hard Boundary Condition. IEEE Transactions on Antennas and Propagation, 68(5), 3807-3814. [8952910].

https://doi.org/10.1109/TAP.2019.2963587

This material is protected by copyright and other intellectual property rights, and duplication or sale of all or part of any of the repository collections is not permitted, except that material may be duplicated by you for your research use or educational purposes in electronic or print form. You must obtain permission for any other use. Electronic or print copies may not be offered, whether for sale or otherwise to anyone who is not an authorised user. 


\title{
Surface Integral Equation Method for Generalized Soft-and-Hard Boundary Condition
}

\author{
Beibei Kong, Pasi Ylä-Oijala, and Ari Sihvola, Fellow, IEEE
}

\begin{abstract}
A surface integral equation (SIE) method is developed for analyzing electromagnetic scattering by objects with generalized soft-and-hard (GSH) boundary conditions. GSH boundary condition is an anisotropic impedance boundary condition, which limits the tangential electric and magnetic fields in specified directions. In the developed SIE formulation, the GSH boundary condition is expressed in a vector form with two sets of orthogonal vectors, which can be combined with the field integral equations. The resulting equation can be discretized with the standard method of moments (MoM) using triangular elements and the Rao-Wilton-Glisson (RWG) functions. By varying the GSH condition the direction of the tangential electric and magnetic fields on the surface can be flexibly changed.
\end{abstract}

Index Terms-Electromagnetic scattering, boundary conditions, generalized soft-and-hard (GSH) boundary, surface integral equation (SIE)

\section{INTRODUCTION}

$\mathbf{E}$ LECTROMAGNETIC scattering by an arbitrarily shaped object is an important and fundamental problem in antenna and microwave engineering. To guarantee a unique solution for the scattered problem, a set of interface conditions is required to relate the fields on both sides of the surface. If the field behavior on the surface of an object can be approximated by a mathematical boundary condition [1], the region of computation can be reduced and the solution of the problem can be significantly simplified. Therefore, boundary conditions are very useful in modeling various real-world problems.

Conventionally, electromagnetic boundary conditions are defined in terms of the tangential components of the fields. The most common examples are the perfect electric and magnetic conductor (PEC and PMC) boundary conditions, which state that the tangential component of the electric or magnetic field vanishes on the boundary, respectively. Impedance boundary condition (IBC) [2], [3] is a generalization of PEC and PMC conditions. Perfect electromagnetic conductor (PEMC) [4] is another type of boundary condition that combines the tangential components of the electric and magnetic fields. It is also possible to express the boundary conditions in terms of the normal field components [5]-[7]. For example, the DB boundary condition requires the normal components of the electric and magnetic flux densities $\boldsymbol{D}$ and $\boldsymbol{B}$ vanish on the surface [8]. By requiring the normal derivatives of the normal components of $\boldsymbol{D}$ and $\boldsymbol{B}$ vanish, the D'B' boundary condition is introduced [9]. The DB and $\mathrm{D}^{\prime} \mathrm{B}^{\prime}$ boundary conditions can be combined to form the mixed IBCs (MIBCs) [10].

The authors are with the Department of Electronics and Nanoengineering, Aalto University, 00076 Espoo, Finland (e-mail: beibei.kong@aalto.fi).
Soft-and-hard (SH) boundary condition is a mathematical idealization of a surface on which both tangential electric and magnetic fields are restricted to one direction [11]. The SH boundary is a convenient model for the tuned corrugated surface, which has been used in various engineering applications such as horn antennas, waveguides, and corner reflectors [12]-[15]. By allowing the electric and magnetic fields to have different tangential directions, the SH boundary can be extended as a more general anisotropic boundary, i.e., the generalized soft-and-hard (GSH) boundary [16].

The surface integral equation (SIE) method is an attractive method for numerical analysis of electromagnetic scattering by arbitrarily shaped targets with boundary conditions. Boundary conditions, such as PEC, PMC and IBC, that can be expressed in terms of the equivalent electric and magnetic surface current densities can be straightforwardly implemented into SIE formulations. Less obvious is the implementation of the boundary conditions expressed in terms of the normal field components [17]-[19]. For anisotropic boundary conditions, like SH condition, the development of the SIE method is even more challenging. Though the SH boundary can be considered as a kind of anisotropic IBC, the impedance dyadic contains either very small or very high values, and the existing numerical methods for anisotropic IBC may not be applicable [20], [21]. In 2006, an SIE method for the SH surface was developed by using rectangular elements and the rooftop functions [22]. In [22], the rectangular elements are oriented to the direction of the corrugation, and the surface currents are automatically restricted to flow in the desired direction. However, since the surface currents are only allowed to flow in the direction of rectangular elements, with this method the direction of the currents can not be flexibly changed and it is not applicable for arbitrary GSH surfaces. To our knowledge, no SIE method exists for GSH so far.

In this paper, we propose an SIE method for the GSH boundary condition. In order to enable modeling of arbitrary GSH surfaces, we use triangular surface elements and the Rao-Wilton-Glisson (RWG) [23] basis and test functions. Numerical experiments are performed to verify the accuracy and efficiency of the proposed method.

\section{Generalized Soft-And-Hard(GSH) Boundary CONDITION}

Before developing the SIE formulation, we first review the definition and the properties of the GSH boundary condition. 


\section{A. Definition of GSH}

Soft-and-hard (SH) condition defines a boundary on which the tangential electric and magnetic fields are ideally conducted in a certain direction. This condition can be written as [15]

$$
\mathbf{v} \cdot \boldsymbol{E}=0, \quad \mathbf{v} \cdot \boldsymbol{H}=0 .
$$

where $\mathbf{v}$ is a tangential vector.

By forcing the tangential electric and magnetic fields to have zero components in two different directions, the $\mathrm{SH}$ boundary can be extended to a generalized Soft-and-Hard (GSH) boundary as [16]

$$
\mathbf{a}_{t} \cdot \boldsymbol{E}=0, \quad \mathbf{b}_{t} \cdot \boldsymbol{H}=0
$$

where $\mathbf{a}_{t}$ and $\mathbf{b}_{t}$ are two arbitrary tangential vectors.

The GSH boundary condition can be written in the form of an anisotropic impedance boundary condition (IBC) over a closed surface $S$ [1], [16]:

$$
\begin{gathered}
\gamma_{t} \boldsymbol{E}=\overline{\bar{Z}}_{s} \cdot \boldsymbol{n} \times \gamma_{t}\left(\eta_{0} \boldsymbol{H}\right) \\
\overline{\bar{Z}}_{s}=\lim _{\delta \rightarrow 0}\left(\delta \mathbf{b}_{t} \mathbf{a}_{t}+\frac{1}{\delta}\left(\mathbf{n} \times \mathbf{a}_{t}\right)\left(\mathbf{n} \times \mathbf{b}_{t}\right)\right) .
\end{gathered}
$$

Here $\boldsymbol{n}$ is the unit normal vector of $S$ and $\eta_{0}$ is the wave impedance of the free space. $\gamma_{t} \boldsymbol{F}=\left.(\boldsymbol{I}-\boldsymbol{n n} \cdot) \boldsymbol{F}\right|_{S}$ denotes tangential component of a vector field on the surface $S$, where $\mathcal{I}\{\boldsymbol{F}\}=\boldsymbol{F}$ is the identity operator.

\section{B. Non-reciprocity and Non-losslessness of GSH}

An impedance boundary is reciprocal if the impedance dyadic satifies $\overline{\bar{Z}}_{s}^{T}=\overline{\bar{Z}}_{s}$ [2]. The impedance boundary is lossless when $\overline{\bar{Z}}_{s}^{T}=-\overline{\bar{Z}}_{s}^{*}$ [2]. Assuming $\mathbf{a}_{t}=\mathbf{x}$ and $\mathbf{b}_{t}=\mathbf{x} \cos \gamma+\mathbf{y} \sin \gamma$, where $\mathbf{x}$ and $\mathbf{y}$ are the local orthogonal tangential unit vectors on $S$ satisfying $\mathbf{x} \times \mathbf{y}=\mathbf{n}$, the surface impedance dyadic (4) can be written in the form

$$
\overline{\bar{Z}}_{s}=\lim _{\delta \rightarrow 0}\left(\mathbf{x} \mathbf{x} \delta \cos \gamma+\mathbf{y} \mathbf{y} \frac{1}{\delta} \cos \gamma+\mathbf{y} \mathbf{x} \sin \gamma\left(\delta-\frac{1}{\delta}\right)\right) .
$$

When $\sin \gamma=0, \mathbf{a}_{t}$ is parallel with $\mathbf{b}_{t}, \overline{\bar{Z}}_{s}$ is symmetric and anti-hermitian, the impedance boundary is reciprocal and lossless. In this case, the conditions of the GSH boundary (2) become conditions of the SH boundary. The GSH boundary is non-reciprocal and non-lossless except in the special case of an SH boundary.

\section{TE/TM Decomposition of Plane Wave}

To analyze the reflection from a GSH boundary, let us split a plane wave $(\boldsymbol{E}, \boldsymbol{H})$ with a propagation direction of a unit vector $\mathbf{u}$ as [1], [16]

$$
(\boldsymbol{E}, \boldsymbol{H})=\left(\boldsymbol{E}_{a}, \boldsymbol{H}_{a}\right)+\left(\boldsymbol{E}_{b}, \boldsymbol{H}_{b}\right)
$$

where

$$
\begin{aligned}
\boldsymbol{E}_{a} & =\frac{1}{\Delta}\left(\mathbf{u} \times \mathbf{a}_{t}\right)\left(\mathbf{b}_{t} \cdot \eta_{0} \boldsymbol{H}_{t}\right) \\
\eta_{0} \boldsymbol{H}_{a} & =\frac{1}{\Delta}\left(\left(\mathbf{a}_{t} \times \mathbf{u}\right) \times \mathbf{u}\right)\left(\mathbf{b}_{t} \cdot \eta_{0} \boldsymbol{H}_{t}\right) \\
\boldsymbol{E}_{b} & =\frac{1}{\Delta}\left(\left(\mathbf{b}_{t} \times \mathbf{u}\right) \times \mathbf{u}\right)\left(\mathbf{a}_{t} \cdot \boldsymbol{E}_{t}\right) \\
\eta_{0} \boldsymbol{H}_{b} & =-\frac{1}{\Delta}\left(\mathbf{u} \times \mathbf{b}_{t}\right)\left(\mathbf{a}_{t} \cdot \boldsymbol{E}_{t}\right)
\end{aligned}
$$

with $\Delta=-\left(\mathbf{u} \times \mathbf{a}_{t}\right) \cdot\left(\mathbf{u} \times \mathbf{b}_{t}\right)$. The decomposed plane waves satisfy $\mathbf{a}_{t} \cdot \boldsymbol{E}_{a}=0, \mathbf{b}_{t} \cdot \boldsymbol{H}_{b}=0$, which are defined as $\mathrm{TE}_{a}$ and $\mathrm{TM}_{b}$ waves, respectively.

Considering a plane wave $\left(\boldsymbol{E}^{i}, \boldsymbol{H}^{i}\right)=\left(\boldsymbol{E}_{a}^{i}, \boldsymbol{H}_{a}^{i}\right)+$ $\left(\boldsymbol{E}_{b}^{i}, \boldsymbol{H}_{b}^{i}\right)$ incident to the GSH boundary, the $\mathrm{TE}_{a}$ wave can be proved to be reflected as a $\mathrm{TE}_{a}$ wave $\left(\boldsymbol{E}_{a}^{r}, \boldsymbol{H}_{a}^{r}\right)$ and the $\mathrm{TM}_{b}$ wave is reflected as a $\mathrm{TM}_{b}$ wave $\left(\boldsymbol{E}_{b}^{r}, \boldsymbol{H}_{b}^{r}\right)$ [1], [16]. It can also be shown that $\mathbf{n} \times\left(\boldsymbol{H}_{a}^{i}+\boldsymbol{H}_{a}^{r}\right)=0$ and $\mathbf{n} \times\left(\boldsymbol{E}_{b}^{i}+\boldsymbol{E}_{b}^{r}\right)=0$. Thus, the GSH boundary behaves as a PMC surface for $\mathrm{TE}_{a}$ wave and a PEC surface for $\mathrm{TM}_{b}$ wave [1], [16]. In other words, the problem of scattering by a GSH boundary can be split into two simpler problems, i.e. PEC for $\mathrm{TM}_{b}$ wave and $\mathrm{PMC}$ for $\mathrm{TE}_{a}$ wave. This property is utilized in the analysis of the numerical results in Section IV.

\section{Reflection from GSH}

The relations between the incident and reflected fields can be defined using reflection dyadics as

$$
\boldsymbol{E}^{r}=\overline{\bar{R}}_{E} \cdot \boldsymbol{E}^{i}, \quad \boldsymbol{H}^{r}=\overline{\bar{R}}_{H} \cdot \boldsymbol{H}^{i}
$$

where the reflection dyadics of the GSH boundary are [7], [16] $\overline{\bar{R}}_{E}=\frac{1}{\Delta}\left[\left(\mathbf{u}^{r} \times \mathbf{a}_{t}\right)\left(\mathbf{u}^{i} \times \mathbf{b}_{t}\right)+\left(\mathbf{u}^{r} \times\left(\mathbf{u}^{r} \times \mathbf{b}_{t}\right)\right)\left(\mathbf{u}^{i} \times\left(\mathbf{u}^{i} \times \mathbf{a}_{t}\right)\right)\right](10)$ $\overline{\bar{R}}_{H}=\frac{1}{\Delta}\left[\left(\mathbf{u}^{r} \times \mathbf{b}_{t}\right)\left(\mathbf{u}^{i} \times \mathbf{a}_{t}\right)+\left(\mathbf{u}^{r} \times\left(\mathbf{u}^{r} \times \mathbf{a}_{t}\right)\right)\left(\mathbf{u}^{i} \times\left(\mathbf{u}^{i} \times \mathbf{b}_{t}\right)\right)\right]$.

The relation between the incident unit direction $\left(\mathbf{u}^{i}\right)$ and the reflected unit direction $\left(\mathbf{u}^{r}\right)$ is $\mathbf{u}^{r}=\overline{\bar{C}} \cdot \mathbf{u}^{i}$, where the mirroring dyadic $\overline{\bar{C}}$ is

$$
\overline{\bar{C}}=\overline{\bar{I}}-2 \boldsymbol{n n}
$$

in which $\overline{\bar{I}}$ is the unit dyadic. Note that $\Delta$ is the same for $\mathbf{u}=\mathbf{u}^{i}$ and $\mathbf{u}=\mathbf{u}^{r}$. We use these analytic reflection dyadics to calculate the surface currents on the illuminated region for the physical optics (PO) method [24], [25], which is employed as a reference to verify our results obtained by the SIE method.

\section{Surface Integral Equation Method FOR GSH}

In this section, we develop a SIE method for electromagnetic scattering by a closed object with GSH boundary conditions.

\section{A. Surface Integral Formulation}

Consider time-harmonic electromagnetic scattering (with time factor $e^{j \omega t}$ ) by a closed non-penetrable object in free space with permittivity $\varepsilon_{0}$ and permeability $\mu_{0}$. On the surface of the object $S$ we assume GSH boundary condition (2). Let $\boldsymbol{J}=\boldsymbol{n} \times \boldsymbol{H}$ and $\boldsymbol{M}=-\boldsymbol{n} \times \boldsymbol{E}$ denote the equivalent electric and magnetic surface current densities on $S$. For a given incident wave $\left(\boldsymbol{E}^{i}, \boldsymbol{H}^{i}\right)$, the tangential field integral equations can be formulated as follows

$$
\left[\begin{array}{cc}
-\gamma_{t} \mathcal{T} & \gamma_{t} \mathcal{K}+\frac{1}{2} \boldsymbol{n} \times \mathcal{I} \\
-\gamma_{t} \mathcal{K}-\frac{1}{2} \boldsymbol{n} \times \boldsymbol{I} & -\gamma_{t} \mathcal{T}
\end{array}\right]\left[\begin{array}{c}
\eta_{0} \boldsymbol{J} \\
\boldsymbol{M}
\end{array}\right]=\left[\begin{array}{c}
\gamma_{t} \boldsymbol{E}^{\mathrm{i}} \\
\eta_{0} \gamma_{t} \boldsymbol{H}^{\mathrm{i}}
\end{array}\right] .
$$

Here the operators $\mathcal{T}$ and $\mathcal{K}$ are defined as

$$
\mathcal{T}\{\boldsymbol{X}\}(\boldsymbol{r})=-j k_{0} \int_{p . v .}\left[1+\frac{1}{k_{0}^{2}} \nabla \nabla \cdot\right] \boldsymbol{X}\left(\boldsymbol{r}^{\prime}\right) G\left(\boldsymbol{r}, \boldsymbol{r}^{\prime}\right) d S^{\prime}
$$




$$
\mathcal{K}\{\boldsymbol{X}\}(\boldsymbol{r})=-\int_{p . v .} \boldsymbol{X}\left(\mathbf{r}^{\prime}\right) \times \nabla G\left(\boldsymbol{r}, \boldsymbol{r}^{\prime}\right) d S^{\prime}
$$

where $k_{0}=\omega \sqrt{\varepsilon_{0} \mu_{0}}, G\left(\boldsymbol{r}, \boldsymbol{r}^{\prime}\right)=e^{-j k_{0} \mid \boldsymbol{r}-\boldsymbol{r}^{\prime}}|/ 4 \pi| \boldsymbol{r}-\boldsymbol{r}^{\prime} \mid$ is the Green's function of free space, and p.v. stands for the principal value integral.

Since the tangential field integral equations are formulated in terms of the equivalent surface electric and magnetic currents, we rewrite the GSH in terms of these currents as

$$
\begin{aligned}
& \mathbf{a}_{t} \cdot \mathbf{n} \times \boldsymbol{M}=0 \\
& \mathbf{b}_{t} \cdot \mathbf{n} \times \boldsymbol{J}=0 .
\end{aligned}
$$

These are scalar equations and thus can be not be directly combined with the SIEs. To allow this, we multiply (16) with two orthogonal vectors $\mathbf{a}_{t}$ and $\mathbf{n} \times \mathbf{a}_{t}$, and multiply (17) with $\mathbf{b}_{t}$ and $\mathbf{n} \times \mathbf{b}_{t}$, as

$$
\begin{array}{cc}
\mathbf{a}_{t}\left(\mathbf{a}_{t} \cdot \mathbf{n} \times \boldsymbol{M}\right)=0, & \left(\mathbf{n} \times \mathbf{a}_{t}\right)\left(\mathbf{a}_{t} \cdot \mathbf{n} \times \boldsymbol{M}\right)=0 \\
\mathbf{b}_{t}\left(\mathbf{b}_{t} \cdot \mathbf{n} \times \boldsymbol{J}\right)=0, & \left(\mathbf{n} \times \mathbf{b}_{t}\right)\left(\mathbf{b}_{t} \cdot \mathbf{n} \times \boldsymbol{J}\right)=0 .
\end{array}
$$

In this way, the GSH boundary conditions are expressed in vector form and can be combined with the SIEs. Let $\mathbf{n} \times \mathbf{a}_{t}=$ $\mathbf{c}_{t}, \mathbf{n} \times \mathbf{b}_{t}=\mathbf{d}_{t}$. Then (18) and (19) can be written as

$$
\begin{aligned}
\left(\mathbf{n} \times \mathbf{c}_{t}\right) \mathbf{c}_{t} \cdot \boldsymbol{M}=0, & \mathbf{c}_{t} \mathbf{c}_{t} \cdot \boldsymbol{M}=0, \\
\left(\mathbf{n} \times \mathbf{d}_{t}\right) \mathbf{d}_{t} \cdot \eta_{0} \boldsymbol{J}=0, & \mathbf{d}_{t} \mathbf{d}_{t} \cdot \eta_{0} \boldsymbol{J}=0 .
\end{aligned}
$$

Combining Eq. (20) with (13), the final SIE formulation for the scattering of GSH boundary is written as

$$
\begin{aligned}
& {\left[\begin{array}{cc}
-\gamma_{t} \mathcal{T}+\mathbf{d}_{t} \mathbf{d}_{t} . & \gamma_{t} \mathcal{K}+\frac{1}{2} \boldsymbol{n} \times \mathcal{I}-\left(\mathbf{n} \times \mathbf{c}_{t}\right) \mathbf{c}_{t} \cdot \\
-\gamma_{t} \mathcal{K}-\frac{1}{2} \boldsymbol{n} \times \mathcal{I}+\left(\mathbf{n} \times \mathbf{d}_{t}\right) \mathbf{d}_{t} . & -\gamma_{t} \mathcal{T}+\mathbf{c}_{t} \mathbf{c}_{t} .
\end{array}\right]} \\
& {\left[\begin{array}{r}
\eta_{0} \boldsymbol{J} \\
\boldsymbol{M}
\end{array}\right]=\left[\begin{array}{c}
\gamma_{t} \boldsymbol{E}^{i} \\
\eta_{0} \gamma_{t} \boldsymbol{H}^{i}
\end{array}\right] .}
\end{aligned}
$$

\section{B. Numerical Strategy}

Next, Eq. (21) is discretized with the method of moments (MoM). Expanding the unknown surface current densities $J$ and $\boldsymbol{M}$ with the RWG basis functions and employing the Galerkin testing method, the integral equations are converted into the following matrix equation

$$
\left[\begin{array}{cc}
-\mathbf{T}+\mathbf{G}^{\mathbf{d}_{t} \mathbf{d}_{t}} & \mathbf{K}+\frac{1}{2} \mathbf{R}^{\overline{\bar{I}}}-\mathbf{R}^{\mathbf{c}_{t} \mathbf{c}_{t}} \\
-\mathbf{K}-\frac{1}{2} \mathbf{R}^{\overline{\bar{I}}}+\mathbf{R}^{\mathbf{d}_{t} \mathbf{d}_{t}} & -\mathbf{T}+\mathbf{G}^{\mathbf{c}_{t} \mathbf{c}_{t}}
\end{array}\right]\left[\begin{array}{c}
\tilde{\mathbf{x}}^{J} \\
\mathbf{x}^{M}
\end{array}\right]=\left[\begin{array}{c}
\mathbf{b}^{E} \\
\tilde{\mathbf{b}}^{H}
\end{array}\right]
$$

with elements

$$
\begin{array}{r}
\mathbf{T}_{i j}=\int_{S} \mathbf{g}_{i} \cdot \mathcal{T}\left\{\mathbf{g}_{j}\right\} d S, \quad \mathbf{K}_{i j}=\int_{S} \mathbf{g}_{i} \cdot \mathcal{K}\left\{\mathbf{g}_{j}\right\} d S \\
\mathbf{R}_{i j}^{\overline{\bar{A}}}=\int_{S}\left(\mathbf{g}_{i} \times \mathbf{n}\right) \cdot \overline{\bar{A}} \cdot \mathbf{g}_{j} d S, \quad \mathbf{G}_{i j}^{\overline{\bar{A}}}=\int_{S} \mathbf{g}_{i} \cdot \overline{\bar{A}} \cdot \mathbf{g}_{j} d S
\end{array}
$$

and excitation vectors

$$
\mathbf{b}_{i}^{E}=\int_{S} \mathbf{g}_{i} \cdot \boldsymbol{E}^{i} d S, \quad \tilde{\mathbf{b}}_{i}^{H}=\eta_{0} \int_{S} \mathbf{g}_{i} \cdot \boldsymbol{H}^{i} d S
$$

Here $\mathrm{g}_{i}$ denotes an RWG function associated with an edge of the mesh. Vectors $\tilde{\mathbf{x}}^{J}$ and $\mathbf{x}^{M}$ include the coefficients of the RWG basis function approximations of $\eta_{0} \boldsymbol{J}$ and $\boldsymbol{M}$, respectively.

In order to allow efficient solutions for large problems, the Multilevel Fast Multipole Algorithm (MLFMA) [26] is employed to speed up the computation of the matrix-vector multiplication. When MLFMA is employed, the matrix is partitioned into near-field and far-field interactions. The terms of the boundary conditions affect only the near-field interactions, while the far-field interactions only relate to $\gamma_{t} \mathcal{T}$ and $\gamma_{t} \mathcal{K}$ operators. Thus the standard MLFMA can be used. This property is one of the main benefits of our formulation.

The near-field matrix of MLFMA is used as a preconditioner to further improve the iteration speed [27]. Writing the matrix equation (22) into a compact form as $\mathbf{M x}=\mathbf{b}$, the preconditioned matrix equation can be expressed as

$$
\left(\mathbf{M}^{N F}\right)^{-1} \mathbf{M} \mathbf{x}=\left(\mathbf{M}^{N F}\right)^{-1} \mathbf{b}
$$

where $\mathbf{M}^{N F}$ is the near-field part of $\mathbf{M}$.

\section{Numerical Results}

This section investigates numerical solutions obtained by the proposed SIE method. In our calculations, the MLMFA with the preconditioner and the generalized minimal residual (GMRES) iterative solver is used unless otherwise noted. The dimension of the Krylov subspace is 100 and the residual error is $10^{-3}$ except for the results in Fig. 4, where value $10^{-5}$ is used.

\section{A. Surface Current Distribution}

First, we calculate scattering by a cube with the GSH boundary condition and study current distributions. The cube has an edge length of $2 \mathrm{~m}$, which is two wavelengths at the operating frequency $300 \mathrm{MHz}$. Fig. 1(a) shows the direction of $\mathbf{a}_{t}$, and the angle between vectors $\mathbf{a}_{t}$ and $\mathbf{b}_{t}$ is denoted by $\gamma$, as shown in Fig. 1(b). The electric field of the incident plane wave is $x$-polarized and propagating along the $-z$ direction. Meshing the cube with a mesh size of $1 / 10$ wavelengths gives 8196 edges. The proposed SIE method provides converged solutions within 10 and 15 iteration steps for $\gamma=0^{\circ}$ and $\gamma=30^{\circ}$, respectively. The surface current distribution of the cube for $\gamma=0^{\circ}$ and $\gamma=30^{\circ}$ are shown in Fig. 2. When $\gamma=0^{\circ}$, the upper surface behaves as PEC since the incident electric field is parallel to $\mathbf{a}_{t}$ and $\mathbf{b}_{t}$. Thus the magnetic currents vanish on the upper surface. Since $\boldsymbol{J}$ is parallel with $\mathbf{b}_{t}$ and $M$ is parallel with $\mathbf{a}_{t}$, the currents satisfy the GSH condition.

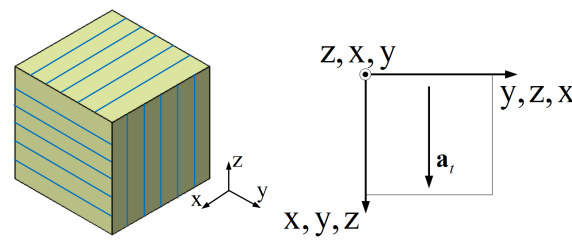

(a)

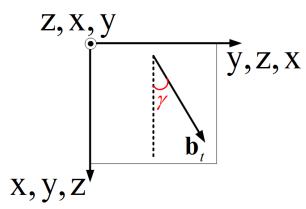

(b)
Fig. 1. A cube with GSH surface. (a) The direction of $\mathbf{a}_{t}$ on the surface of the cube. (b) The direction of $\mathbf{a}_{t}$ and $\mathbf{b}_{t}$ on each face of the cube. 


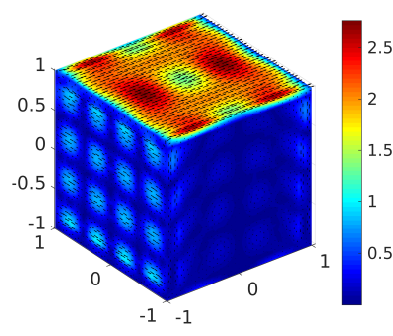

(a)

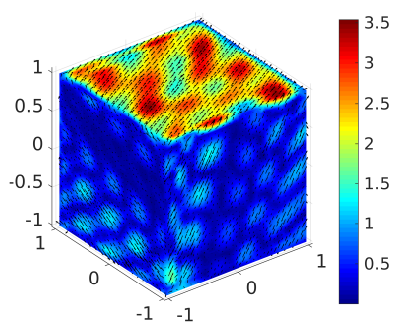

(c)

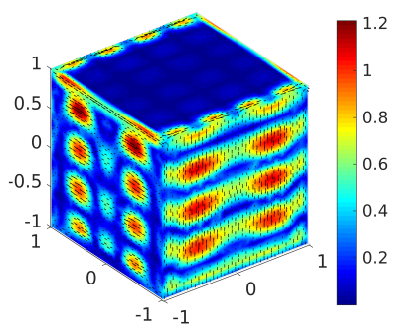

(b)

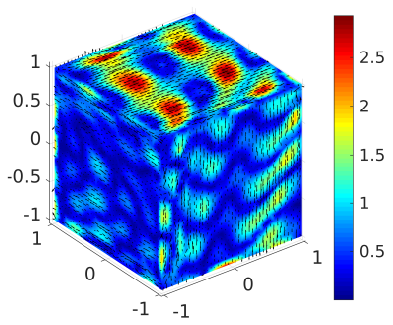

(d)
Fig. 2. The surface current distributions of a GSH cube with an edge length of $2 \mathrm{~m}$ at a frequency of $300 \mathrm{MHz}$. The direction of $\mathbf{a}_{t}$ and $\mathbf{b}_{t}$ are shown in Fig. 1. (a) $\operatorname{Real}\left\{\eta_{0} \boldsymbol{J}\right\}$ at $\gamma=0^{\circ}$. (b) $\operatorname{Real}\{\boldsymbol{M}\}$ at $\gamma=0^{\circ}$. (c) $\operatorname{Real}\left\{\eta_{0} \boldsymbol{J}\right\}$ at $\gamma=30^{\circ}$. (d) $\operatorname{Real}\{\boldsymbol{M}\}$ at $\gamma=30^{\circ}$.

\section{B. Non-reciprocity of GSH}

We next verify the non-reciprocal character of the GSH boundary. We calculate scattering by the cube shown in Fig. 1 . When $\mathbf{a}_{t}$ and $\mathbf{b}_{t}$ are parallel, the boundary is reciprocal and it corresponds to the SH boundary. Otherwise, the boundary is the GSH boundary and is non-reciprocal. We calculate the bistatic radar cross-section (RCS) of the cube with a $300 \mathrm{MHz}$ plane wave incident from $\theta=45^{\circ}$ and $\phi=45^{\circ}$. Both $\theta$ - and $\phi$-polarization of the incident electric fields are considered. The bistatic RCS for $\gamma=0^{\circ}$ and $\gamma=30^{\circ}$ in the $x-y$ plane are plotted with solid lines in Fig. 3(a) and (b), respectively. In Fig. 3, $\phi=0^{0}$ and $\phi=90^{\circ}$ correspond to the $x$ and $y$ direction, respectively.

Then we interchange the incident and observation direction, i.e., the incident angles lie in the $x-y$ plane and the observation angle is $\theta=45^{\circ}$ and $\phi=45^{\circ}$. The bistatic RCS are calculated and drawn with dashed lines in Fig. 3. It can be observed that when $\gamma=0^{\circ}$, the interchange of the incident and observation direction give identical results. This verifies that the system is reciprocal for the SH boundary. The results for $\gamma=30^{\circ}$ are different when the incident and observation directions are interchanged, which shows the non-reciprocity of GSH boundary with non-parallel $\mathbf{a}_{t}$ and $\mathbf{b}_{t}$.

\section{Further Analysis of the Numerical Performance}

In our calculations, the proposed SIE method is found to have a slow iteration convergence speed when the angle between $\mathbf{a}_{t}$ and $\mathbf{b}_{t}$ is large. As an example, the number of iterations for a cube of an edge length $1 \mathrm{~m}$ at a frequency of $300 \mathrm{MHz}$ is plotted in Fig. 4. The results are obtained using MoM with a GMRES iterative solver and without any preconditioner. On the surface of the cube, the vectors $\mathbf{a}_{t}$ and $\mathbf{b}_{t}$ are defined as shown in Fig. 1. The surface mesh of the cube contains 2016 edges with the mesh size of $1 / 10$ wavelengths. The electric field of the incident plane wave is $x$-polarized and propagating along the $-z$ direction. The condition number of

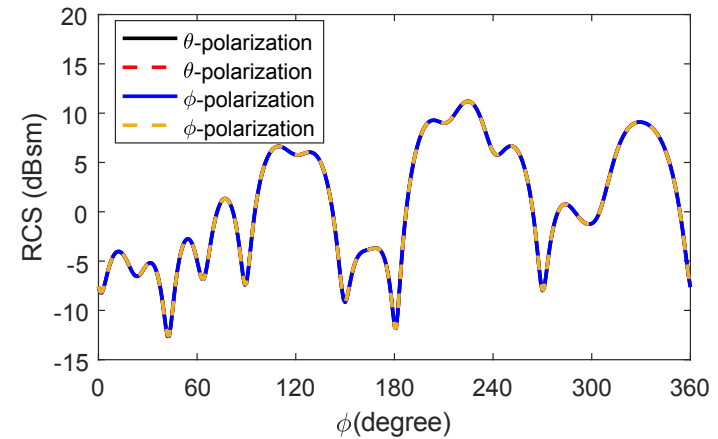

(a)

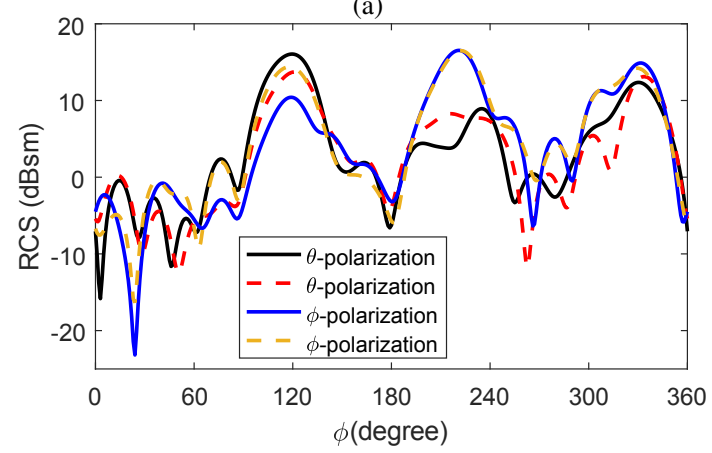

(b)

Fig. 3. Bistatic RCS for the GSH cube with an edge length of $2 \mathrm{~m}$ at a frequency of $300 \mathrm{MHz}$. The direction of $\mathbf{a}_{t}$ and $\mathbf{b}_{t}$ are shown in Fig. 1. (a) $\gamma=0^{\circ}$. (b) $\gamma=30^{\circ}$.

the matrix $\mathbf{M}$ is also calculated. As can be seen from Fig. 4, both the number of iterations and condition number of the matrix increase dramatically as $\gamma$ is increased.

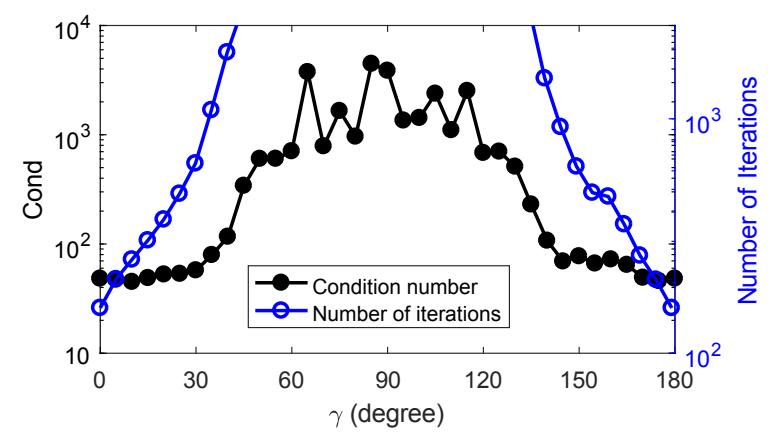

Fig. 4. The number of iterations and the condition number for a cube with an edge length of $1 \mathrm{~m}$ at $300 \mathrm{MHz}$. On the surface of the cube, GSH is assumed as $\mathbf{a}_{t}$ and $\mathbf{b}_{t}$ are defined as in Fig. 1.

To find reasons for these observations, let us analyze the reflection dyadics (10)-(11). In these dyadics, $\Delta$ can be rewritten as

$$
\Delta=-\left(\mathbf{u} \times \mathbf{a}_{t}\right) \cdot\left(\mathbf{u} \times \mathbf{b}_{t}\right)=\frac{k_{t}^{2}}{k_{0}^{2}} \mathbf{a}_{t} \cdot \mathbf{u}_{t} \mathbf{b}_{t} \cdot \mathbf{u}_{t}-\mathbf{a}_{t} \cdot \mathbf{b}_{t}
$$

where $k_{0}$ is the free space wavenumber, the unit vector $\mathbf{u}_{t}$ denotes the direction of the tangential component of $\mathbf{u}$ and satisfies $\mathbf{u}=\frac{k_{t}}{k_{0}} \mathbf{u}_{t} \pm \frac{k_{n}}{k_{0}} \mathbf{n}$. When $\Delta=0$, the magnitude of the reflection dyadics of the GSH boundary becomes infinite. This is the situation of a matched wave [1], [7], where the reflected wave by itself already satisfies the boundary condition. The condition of the matched wave can be obtained from (26) as

$$
k_{t}^{2}=k_{0}^{2} \frac{\mathbf{a}_{t} \cdot \mathbf{b}_{t}}{\mathbf{a}_{t} \cdot \mathbf{u}_{t} \mathbf{b}_{t} \cdot \mathbf{u}_{t}}
$$


Assuming $\mathbf{a}_{t}=\mathbf{x} \cos (\gamma / 2)-\mathbf{y} \sin (\gamma / 2), \mathbf{b}_{t}=\mathbf{x} \cos (\gamma / 2)+$ $\mathbf{y} \sin (\gamma / 2)$ and $\mathbf{u}_{t}=\mathbf{x} \cos \phi+\mathbf{y} \sin \phi$, the dispersion equation (27) can be expressed as

$$
\frac{k_{t}^{2}}{k_{0}^{2}}=\frac{\cos \gamma}{\cos (\gamma / 2-\phi) \cos (\gamma / 2+\phi)}
$$

In Fig. 5 we plot $k_{t}^{2} / k_{0}^{2}$ given by (28) as a function of $\phi$ for different values of $\gamma$. We observe that when $\gamma=0^{\circ}, k_{t}^{2} / k_{0}^{2}$ is larger than one, excluding the case when $\mathbf{u}_{t}$ is parallel with both $\mathbf{a}_{t}$ and $\mathbf{b}_{t}$, i.e, as $\phi=0$ (see Fig. 5(b)). For values larger than one, a real matched wave can never emerge. As $\gamma$ increases from $0^{\circ}$ to $90^{\circ}$, the range of $\phi$ corresponding to $k_{t}^{2} \leq k_{0}^{2}$ increases, i.e., the matched wave is more likely to appear. The singularity of the matched wave (in which case the value of reflection coefficient explodes) is most probably one reason for the numerical problem indicated in Fig. 4.

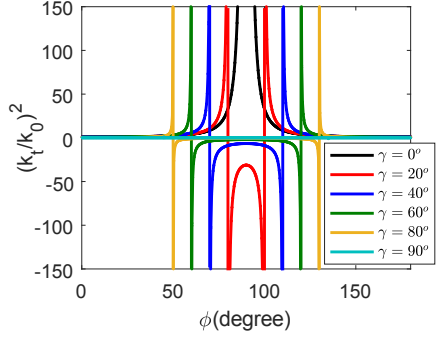

(a)

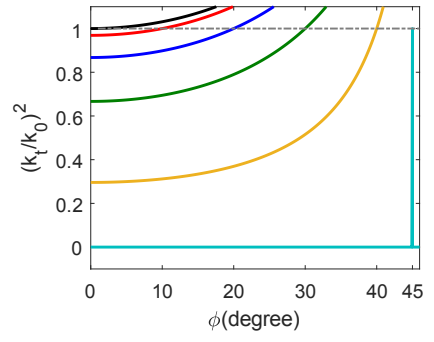

(b)
Fig. 5. (a) The value of $k_{t}^{2} / k_{0}^{2}$ given by (28) as a function of $\phi$ as $\gamma$ is varied from $0^{\circ}$ to $90^{\circ}$. (b) Local zoom of Fig. 5(a).

Another aspect is the absorption behavior. For that we calculate the scattering efficiency $Q_{\text {sca }}$, absorption efficiency $Q_{\mathrm{abs}}$, and the extinction efficiency $Q_{\text {ext }}$ [28] of the cube at a frequency of $300 \mathrm{MHz}$. The electric field of the incident plane wave is $x$-polarized and propagating along the $-z$ direction. The results are calculated using MoM with a direct solver. As shown in Fig. 6, the scattering efficiency obtains very high values as $\gamma$ approaches $90^{\circ}$. Within that region, $Q_{\text {sca }}$ is very sensitive on $\gamma$, i.e., small changes on $\gamma$ can lead to high changes on $Q_{\text {sca. }}$. In this kind of situation, numerical methods usually lose their accuracy and efficiency, as was demonstrated in [28] in the case of impedance boundary. Also the computed $Q_{\text {ext }}$ shows very high error within the same region.

The absorption efficiency, defined as $Q_{\mathrm{abs}}=Q_{\text {ext }}-Q_{\text {sca }}$, is negative, which means that the surface is active. The high value of $\left|Q_{\text {abs }}\right|$ indicates the system is unstable in this region.

\section{Comparison with PO Method}

As last examples, we simulate three electrically large targets with the proposed SIE method. The results are verified with the Physical Optics (PO) method. The TE/TM decomposition is used to analyze and further verify the accuracy of our results.

1) SH Trihedral Corner Reflector: First, we consider trihedral corner reflectors TCR1 and TCR2 with the SH surface. The trihedral corner reflectors are constructed using three isosceles right triangles with a cathetus length of $1.8 \mathrm{~m}$ and a thickness of $30 \mathrm{~mm}$. The directions of corrugations on the surfaces of TCR1 and TCR2 are shown in Fig. 7. The monostatic RCS of the reflectors are calculated at a frequency of $1 \mathrm{GHz}$. The mesh size is 0.1 wavelengths and the number of

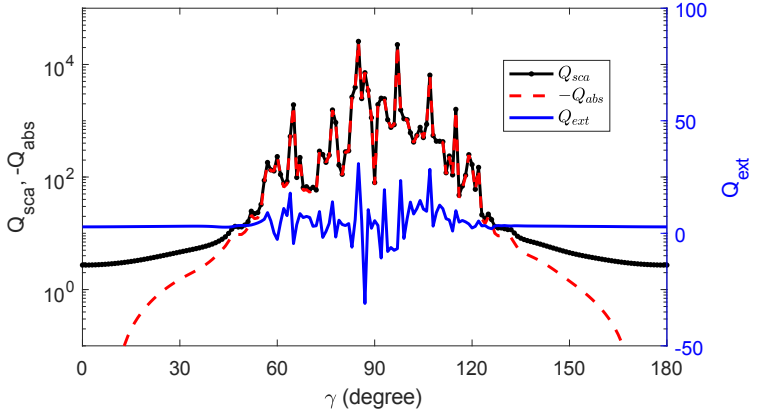

Fig. 6. $Q_{\mathrm{sca}},-Q_{\mathrm{abs}}$ and $Q_{\text {ext }}$ of a cube with an edge length of $1 \mathrm{~m}$ at a frequency of $300 \mathrm{MHz}$. On the surface of the cube, GSH is assumed as $\mathbf{a}_{t}$ and $\mathbf{b}_{t}$ are defined as in Fig. 1.

edges is 39417 . The incident field is right-handedly circularly polarized with the incident polar angle $\theta=54.74^{\circ}$ and the azimuth angle $\phi$ is varied from $0^{\circ}$ to $90^{\circ}$. The results are normalized with respect to the PEC trihedral corner so that the $0 \mathrm{~dB}$ corresponds to the maximum RCS value of the PEC reflector, as shown in Fig. 8. Since the SH surface retains the handedness of the field, the cross-polarized response of TCR1 and TCR 2 are both zero. For TCR1, the co-polarized response has the minimum value when $\phi=45^{\circ}$, which is the same with the results in [22]. Compared with TCR1, TCR2 has a very different co-polarized RCS, which shows that the direction of the corrugation will hiohly affect the hackscattering response.

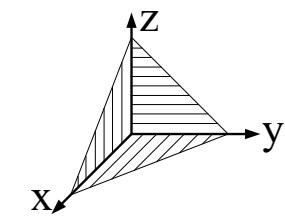

TCR1

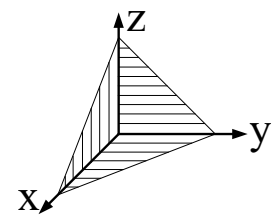

TCR2
Fig. 7. TCR1 and TCR2, trihedral corner reflectors with SH surfaces. The corrugation on the surfaces is parallel with the principal axes.

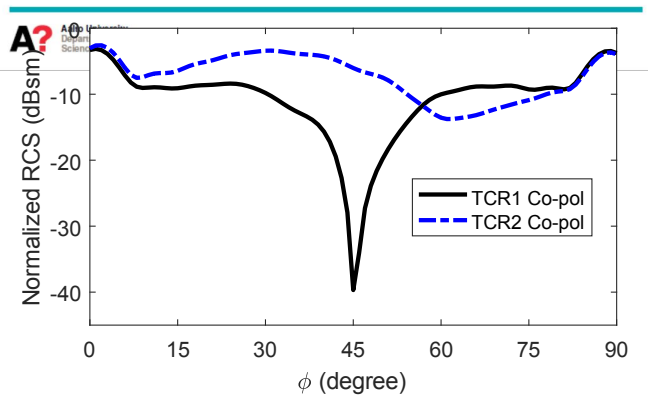

Fig. 8. The normalized monostatic RCS of TCR1 and TCR2.

2) SH Cylinder: Then, we consider a cylinder with an $\mathrm{SH}$ surface. The direction of corrugation on the side, and the ends of the cylinder, are parallel with the $z$ and $x$ axis, respectively, as shown in Fig. 9(a). The radius of the cylinder is $1.5 \mathrm{~m}$ and the height is $3 \mathrm{~m}$. The cylinder is illuminated by a plane wave at a frequency of $600 \mathrm{MHz}$ with a $\phi$-polarized incident electric field. Since the incident electric field is perpendicular to the corrugation on the lateral surface of the cylinder, that part of the cylinder will behave as a PMC surface. Similarly, the ends of the cylinder will also behave as a PMC surface when the incident direction of the plane wave is parallel with the $x-z$ 
plane. In addition, if the incident direction of the plane wave is parallel with the $y-z$ plane, the ends of the cylinder will behave as a PEC surface.

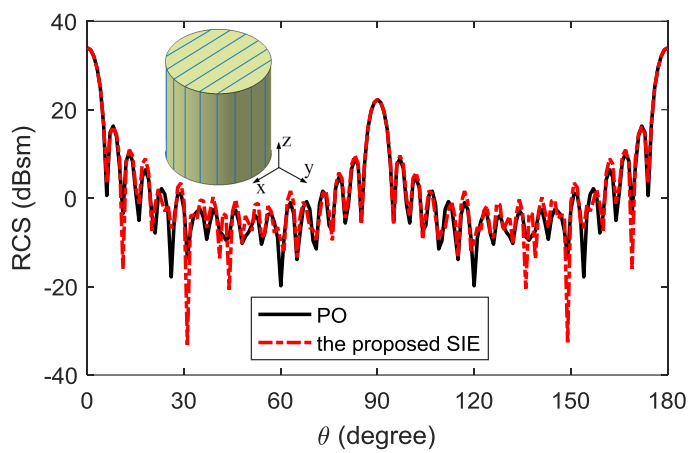

(a)

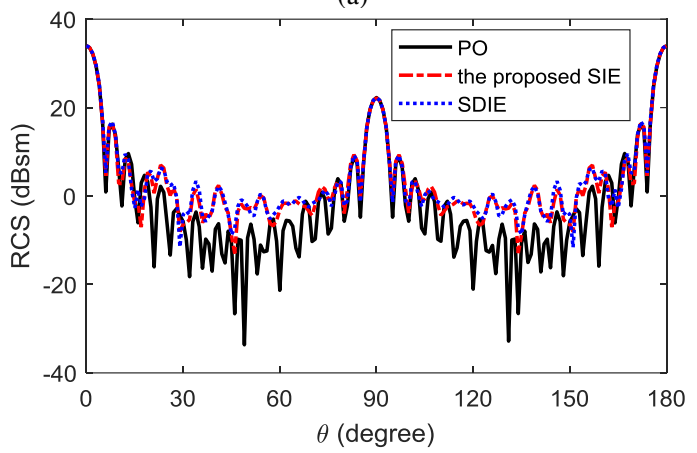

(b)

Fig. 9. Monostatic RCS for an SH cylinder with a radius of $1.5 \mathrm{~m}$ and a height of $3 \mathrm{~m}$ at a frequency of $600 \mathrm{MHz}$. (a) The incident and observation angles lie in the $x-z$ plane. Insert: a cylinder with corrugated surface with the direction of corrugation on the side and the ends parallel with $z$ axis and $x$ axis. (b) The incident and observation angles lie in the $y$ - $z$ plane.

We calculate the monostatic RCS of the cylinder in both $x-z$ plane and $y-z$ plane. The cylinder is meshed with a mesh size of $1 / 10$ wavelengths and the number of edges is 61002. The incident direction is varied form $\theta=0^{\circ}(-z$ direction) to $\theta=180^{\circ}$ ( $z$ direction) with a step of $1^{\circ}$. The average number of iterations of the GMRES solver is 52 . The results are plotted in Fig. 9 and compared with the results of the PO method [24], [25]. Though the PO method does not consider the edge diffractions, the results in the $x$ - $z$ plane obtained by the proposed SIE method and the PO method are in good agreement. Clearly, the disagreement between the results increases for the oblique incidence in the $y$ - $z$ plane. In this case, the side and the ends of the cylinder behave as different kind of surfaces and the boundary condition is discontinuous at the edges. The lack of edge diffraction makes the accuracy of the PO worse for grazing incidence.

To further verify our results in the $y-z$ plane, the cylinder is simulated with the self-dual integral equation (SDIE) method [29] with isotropic IBC. In the SDIE method, the normalized surface impedance on the ends of the cylinder is set as $10^{-5}$ (imitating PEC) and that on the side surface is set as $10^{5}$ (imitating PMC) [30]. As can be observed from Fig. 9 (b), the agreement between the results of the SDIE method and the method proposed in this paper is good.

3) GSH Plate: We consider next a large square plate with an edge length of $10 \mathrm{~m}$ and thickness of $0.02 \mathrm{~m}$, as shown in Fig. 10 (a). The surface of the plate is equipped with the GSH boundary. The directions of $\mathbf{a}_{t}$ and $\mathbf{b}_{t}$ on the top and bottom surfaces are the same as in Fig. 1 (b). On the side surfaces vectors $\mathbf{a}_{t}$ and $\mathbf{b}_{t}$ are parallel to the $z$ axis. The plate is illuminated by a plane wave at a frequency of 300 $\mathrm{MHz}$ propagating along the $-z$ direction. The polarization of the incident electric field is shown in Fig. 10 (b). The mesh size is $1 / 10$ wavelengths and the number of edges is 70332 . We calculate the monostatic RCS of the plate under different polarization of the incident plane wave. The angle $\phi$ increases from $0^{\circ}$ to $360^{\circ}$ with a step of $1^{\circ}$. The average number of iterations for $\gamma=0^{\circ}, \gamma=20^{\circ}$ and $\gamma=30^{\circ}$ are 62,67 and 54 , respectively. The co- and the cross-polarized monostatic RCS of the plate obtained by the SIE method are compared with the results of the PO [24], [25] in Fig. 11. It can be observed that our SIE results agree well with the results of the PO, which is well suited for scattering from this kind of large planar structures.

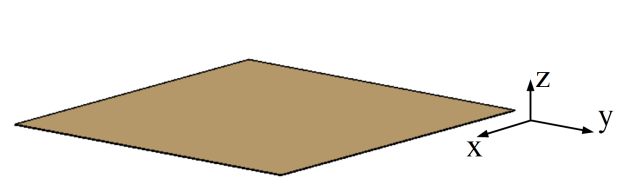

(a)

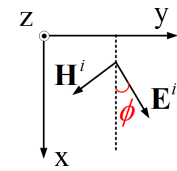

(b)
Fig. 10. A GSH square plate with an edge length of $10 \mathrm{~m}$ and thickness of $0.02 \mathrm{~m}$ illuminated by a normally incident plane wave. (a) The location of the square plate in the rectangular coordinate system. (b) The polarization of the incident plane wave.

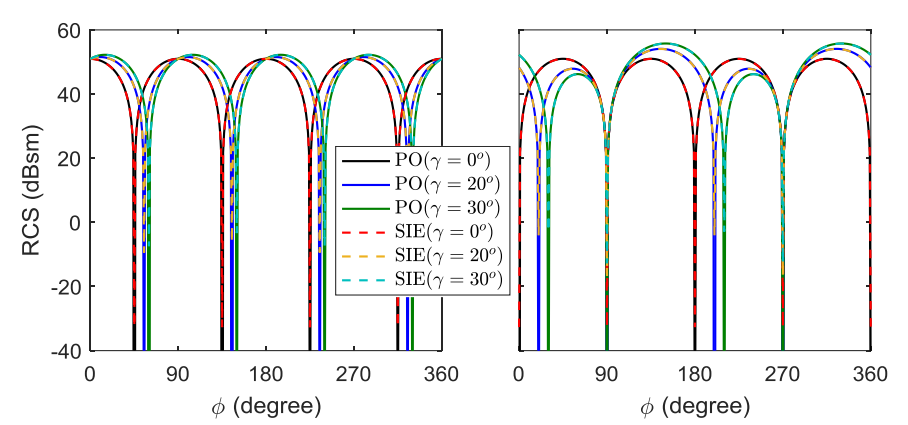

(a)

(b)

Fig. 11. Monostatic RCS of the plate in Fig. 10 at a frequency of $300 \mathrm{MHz}$. (a) Co-polarized RCS. (b) Cross-polarized RCS.

Let us next analyze the numerical results using the TE/TM decomposition of the incident plane wave on the GSH surface. Let the unit vectors $\mathbf{e}$ and $\mathbf{h}$ denote the direction of $\boldsymbol{E}^{i}$ and $\boldsymbol{H}^{i}$. Then $\mathbf{a}_{t}$ and $\mathbf{b}_{t}$ on the upper and lower surfaces of the plate can be written as

$$
\begin{aligned}
& \mathbf{a}_{t}=\mathbf{e} \cos \phi+\mathbf{h} \sin \phi \\
& \mathbf{b}_{t}=\mathbf{e} \cos (\phi-\gamma)+\mathbf{h} \sin (\phi-\gamma) .
\end{aligned}
$$

According to Eqs. (7) and (8), the incident plane wave can be decomposed as follows

$$
\begin{gathered}
\boldsymbol{E}_{a}=\frac{\eta_{0} \boldsymbol{H}^{i} \cdot \mathbf{h}}{\Delta} \sin (\phi-\gamma)(-\mathbf{e} \sin \phi+\mathbf{h} \cos \phi) \\
\eta_{0} \boldsymbol{H}_{a}=\frac{\eta_{0} \boldsymbol{H}^{i} \cdot \mathbf{h}}{\Delta} \sin (\phi-\gamma)(-\mathbf{e} \cos \phi-\mathbf{h} \sin \phi)
\end{gathered}
$$




$$
\begin{aligned}
& \boldsymbol{E}_{b}=\frac{\boldsymbol{E}^{i} \cdot \mathbf{e}}{\Delta} \cos \phi[-\mathbf{e} \cos (\phi-\gamma)-\mathbf{h} \sin (\phi-\gamma)] \\
& \eta_{0} \boldsymbol{H}_{b}=\frac{\boldsymbol{E}^{i} \cdot \mathbf{e}}{\Delta} \cos \phi[\mathbf{e} \sin (\phi-\gamma)-\mathbf{h} \cos (\phi-\gamma)]
\end{aligned}
$$

with $\Delta=-\cos \gamma$. The amplitude of the incident field satisfies $\boldsymbol{E}^{i} \cdot \mathbf{e}=\eta_{0} \boldsymbol{H}^{i} \cdot \mathbf{h}$. For a $\mathrm{TE}_{a}$ wave, the GSH surface behaves as a PMC, for which the reflection dyadic of the electric field is $\overline{\bar{C}}$ (given by Eq. (12)). For a $\mathrm{TM}_{b}$ wave, the GSH surface behaves as a PEC and the reflection dyadic of the electric field is $-\bar{C}$. Thus, the reflection from the GSH surface is

$$
\begin{aligned}
\boldsymbol{E}^{r} & =\overline{\bar{C}} \cdot \boldsymbol{E}_{a}-\overline{\bar{C}} \cdot \boldsymbol{E}_{b} \\
& =\frac{\boldsymbol{E}^{i} \cdot \mathbf{e}}{\Delta}[\mathbf{e} \cos (2 \phi-\gamma)+2 \mathbf{h} \sin (\phi-\gamma) \cos \phi] .
\end{aligned}
$$

From Eq. (32) we can conclude that the co-polarized response has $\cos (2 \phi-\gamma)$ dependence on $\phi$ and the crosspolarized one has $\sin (\phi-\gamma) \cos \phi$ dependence, which are consistent with our results.

4) GSH Cube: As a last example, we calculate scattering from a cube with an edge length of $6 \mathrm{~m}$. The incident direction of the plane wave is parallel with the $x-z$ plane and the electric field is $\theta$-polarized, as shown in Fig. 12(a). The operating frequency is $300 \mathrm{MHz}$. On the two surfaces parallel with the incident plane ( $x-z$ plane), $\mathbf{a}_{t}$ and $\mathbf{b}_{t}$ are parallel to the $z$ axis. The direction of $\mathbf{a}_{t}$ and $\mathbf{b}_{t}$ on the other four surfaces are shown in Fig. 12(b).

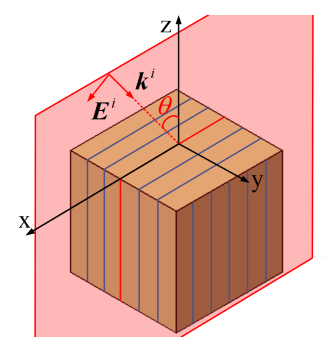

(a)

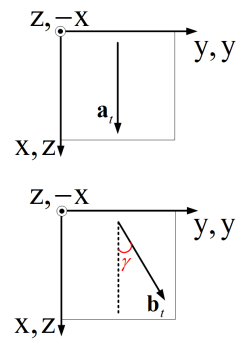

(b)
Fig. 12. A GSH cube with an edge length of $6 \mathrm{~m}$ illuminated by a plane wave. (a) The incident direction and the polarization of the plane wave. (b) The direction of $\mathbf{a}_{t}$ and $\mathbf{b}_{t}$ on each face of the cube.

To get further insight into the simulation results, let us first analyze the response of the surfaces that are in the "bright region" of the PO method. Taking (e $=\boldsymbol{E}^{i} /\left|\boldsymbol{E}^{i}\right|, \mathbf{h}=$ $\left.\boldsymbol{H}^{i} /\left|\boldsymbol{H}^{i}\right|, \mathbf{u}^{i}\right)$ as a rectangular coordinate system, vectors $\mathbf{a}_{t}$ and $\mathbf{b}_{t}$ on the two surfaces that are parallel to the $x-y$ plane can be written as

$$
\begin{aligned}
& \mathbf{a}_{t}=\mathbf{e} \cos \theta-\mathbf{u}^{i} \sin \theta \\
& \mathbf{b}_{t}=\mathbf{e} \cos \gamma \cos \theta-\mathbf{h} \sin \gamma-\mathbf{u}^{i} \cos \gamma \sin \theta .
\end{aligned}
$$

Inserting (33) in (7) and (8) yields the TE/TM decomposition of the incident plane wave

$$
\begin{aligned}
& \boldsymbol{E}_{a}=\left(\eta_{0} \boldsymbol{H}^{i} \cdot \mathbf{h}\right)\left(\frac{\tan \gamma}{\cos \theta} \mathbf{h}\right) \\
& \eta_{0} \boldsymbol{H}_{a}=\left(\eta_{0} \boldsymbol{H}^{i} \cdot \mathbf{h}\right)\left(-\frac{\tan \gamma}{\cos \theta} \mathbf{e}\right) \\
& \boldsymbol{E}_{b}=\left(\boldsymbol{E}^{i} \cdot \mathbf{e}\right)\left(\mathbf{e}-\frac{\tan \gamma}{\cos \theta} \mathbf{h}\right) \\
& \eta_{0} \boldsymbol{H}_{b}=\left(\boldsymbol{E}^{i} \cdot \mathbf{e}\right)\left(\frac{\tan \gamma}{\cos \theta} \mathbf{e}+\mathbf{h}\right) .
\end{aligned}
$$

Thus, the reflection of the electric field on the upper and lower surfaces of the cube is

$$
\boldsymbol{E}^{r}=\overline{\bar{C}} \cdot \boldsymbol{E}_{a}-\overline{\bar{C}} \cdot \boldsymbol{E}_{b}=\left(\boldsymbol{E}^{i} \cdot \mathbf{e}\right)\left(-\mathbf{e}+2 \frac{\tan \gamma}{\cos \theta} \mathbf{h}\right) .
$$

Analogously, the reflection of the electric field on the surfaces parallel to the $x-z$ plane can be expressed as

$$
\boldsymbol{E}^{r}=\overline{\bar{C}} \cdot \boldsymbol{E}_{a}-\overline{\bar{C}} \cdot \boldsymbol{E}_{b}=\left(\boldsymbol{E}^{i} \cdot \mathbf{e}\right)\left(-\mathbf{e}-2 \frac{\tan \gamma}{\cos \theta} \mathbf{h}\right) .
$$

From Eqs. (36) and (37) we can conclude that the crosspolarization response will increase as $\gamma$ increases. When $\gamma$ increases from $\gamma_{1}$ to $\gamma_{2}$, the magnitude of the cross-polarized scattered field will increase by a factor of $\tan \gamma_{2} / \tan \gamma_{1}$. The co-polarization response is independent on the variation of $\gamma$.

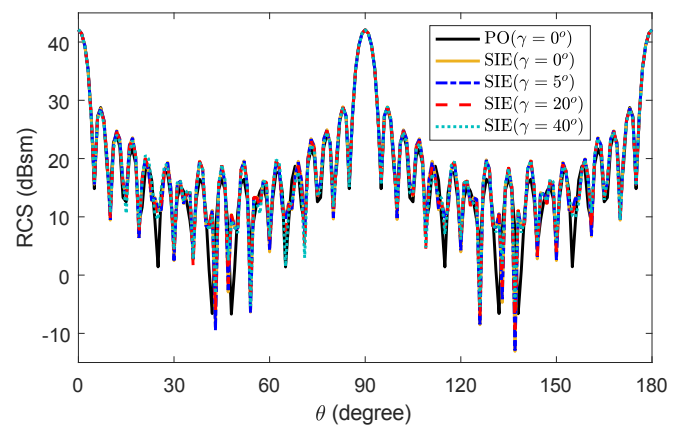

(a)

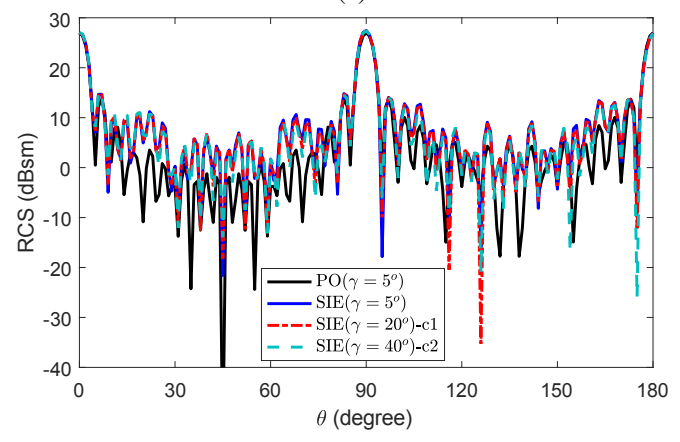

(b)

Fig. 13. Monostatic RCS of the cube in Fig. 12 at a frequency of $300 \mathrm{MHz}$. (a) The co-polarized RCS. (b) The cross-polarized RCS.

Meshing the cube with mesh size of $1 / 10$ wavelengths gives 74526 edges. The monostatic RCS of the cube is calculated as the incident angle $\theta$ is increased from $0^{\circ}$ to $180^{\circ}$. The angular resolution of $\theta$ is $1^{\circ}$. The average number of iterations for $\gamma=0^{\circ}, \gamma=5^{\circ}, \gamma=20^{\circ}$ and $\gamma=40^{\circ}$ are 52, 53, 63, and 119, respectively. The co- and crosspolarized RCS are plotted in Fig. 13(a) and (b), respectively. In Fig. 13(b) $c 1=20 \log _{10}\left(\tan 20^{\circ} / \tan 5^{\circ}\right) \mathrm{dB}$ and $c 2=$ $20 \log _{10}\left(\tan 40^{\circ} / \tan 5^{\circ}\right) \mathrm{dB}$. It can be observed that the copolarized RCS is the same for different values of $\gamma$. The results obtained by the SIE method agree well with the PO results, especially near the normal incidence. As $\gamma$ increases, the cross-polarized RCS increases by the same amount as we analyzed above. As can be seen in Eqs. (36) and (37), the cross-polarized reflection coefficient changes sign on different surfaces. Since PO does not take into account the interaction of different surfaces which have discontinuous boundary conditions, the results of PO have more disagreement with the results of SIE at oblique incidence. 


\section{CONCLUSION}

In this paper we develop a surface integral equation (SIE) method for electromagnetic scattering by arbitrarily shaped 3D objects with generalized soft-and-hard (GSH) boundary conditions. We investigate properties of GSH boundaries, such as non-reciprocity, with both analytical and numerical experiments. In the numerical solution, the GSH conditions are expressed in a vector form. This allows us to combine these conditions with the field integral equations and discretization with standard MoM using triangular elements and RWG functions, as well as straightforward application of MLFMA. Numerical experiments show that the developed SIE method performs well as the angle between the directions of the tangential electric and magnetic fields, specified by the GSH condition, is small enough. As this angle is increased, particularly when it is close to $90^{\circ}$, the convergence speed of the iterative solution of the matrix equation and accuracy of the solution are found to be dramatically reduced. Our analysis indicates that possible reasons for these problems are the appearance of matched waves and the active character of the surface.

\section{REFERENCES}

[1] I. V. Lindell and A. Sihvola, Boundary Conditions in Electromagnetics. Wiley-IEEE Press (To appear).

[2] I. V. Lindell, Methods for Electromagnetic Field Analysis, 2nd ed. New York, NY, USA: Oxford University Press, 1995.

[3] M. A. Leontovitch, "Approximate boundary conditions for the electromagnetic field on the surface of a good conductor," in Investigations Radiowave Propagation Part II. Moscow Russia: Printing House of the Academy of Sciences, 1948.

[4] I. V. Lindell and A. H. Sihvola, "Perfect electromagnetic conductor," $J$. Electromagn. Waves Appl., vol. 19, no. 7, pp. 861-869, Jan. 2005.

[5] V. Rumsey, "Some new forms of Huygens' principle," IRE Trans. Antennas Propag., vol. 7, no. 5, pp. 103-116, Dec. 1959.

[6] A. D. Yaghjian and S. Maci, "Alternative derivation of electromagnetic cloaks and concentrators," New J. Phys., vol. 10, no. 11, p. 115022, Nov. 2008.

[7] I. V. Lindell and A. Sihvola, "Electromagnetic wave reflection from boundaries defined by general linear and local conditions," IEEE Trans. Antennas Propag., vol. 65, no. 9, pp. 4656-4663, Sep. 2017.

[8] I. V. Lindell and A. H. Sihvola, "Electromagnetic boundary and its realization with anisotropic metamaterial," Phys. Rev. E, vol. 79, no. 2, Feb. 2009.

[9] I. V. Lindell and A. Sihvola, "Electromagnetic boundary conditions defined in terms of normal field components," IEEE Trans. Antennas Propag., vol. 58, no. 4, pp. 1128-1135, Apr. 2010.

[10] H. Wallén, I. V. Lindell, and A. Sihvola, "Mixed-impedance boundary conditions," IEEE Trans. Antennas Propag., vol. 59, no. 5, pp. 15801586, May 2011.

[11] P.-S. Kildal, "Definition of artificially soft and hard surfaces for electromagnetic waves," Electron. Lett., vol. 24, no. 3, pp. 168-170, 1988.

[12] E. Lier, "Review of soft and hard horn antennas, including metamaterialbased hybrid-mode horns," IEEE Antennas and Propagation Magazine, vol. 52, no. 2, pp. 31-39, Apr. 2010
[13] A. Macikunas and S. Haykin, "Trihedral twist-grid polarimetric reflector," IEE Proc. F., vol. 140, no. 4, pp. 216-222, 1993.

[14] I. A. Eshrah and A. A. Kishk, "Electric-type dyadic Green's functions for a corrugated rectangular metaguide based on asymptotic boundary conditions," IEEE Trans. Antennas Propag., vol. 55, no. 2, pp. 355-363, Feb. 2007.

[15] I. V. Lindell and P. P. Puska, "Reflection dyadic for the soft and hard surface with application to the depolarising corner reflector," Proc. Inst. Elect. Eng. Micro. Ant. Propag., vol. 143, no. 5, p. 417-421, 1996.

[16] I. V. Lindell, "Generalized soft-and-hard surface," IEEE Trans. Antennas Propag., vol. 50, no. 7, pp. 926-929, Jul. 2002.

[17] J. Markkanen, P. Ylä-Oijala, and A. Sihvola, "Computation of scattering by DB objects with surface integral equation method," IEEE Trans. Antennas Propag., vol. 59, no. 1, pp. 154-161, Jan. 2011.

[18] S. P. Kiminki, J. Markkanen, and P. Ylä-Oijala, "Integral equation solution for the D'B' boundary condition," IEEE Antennas Wireless Propag. Lett., vol. 9, pp. 526-529, 2010.

[19] P. Ylä-Oijala, S. P. Kiminki, H. Wallén, and A. Sihvola, "Uniform surface integral equation formulation for mixed impedance boundary conditions," IEEE Trans. Antennas Propag., vol. 63, no. 12, pp. 57185726, Dec. 2015.

[20] A. Glisson, M. Orman, F. Falco, and D. Koppel, "Electromagnetic scattering by an arbitrarily shaped surface with an anisotropic impedance boundary condition," ACES Journal, vol. 10, no. 3, pp. 93-106, 1995.

[21] K. Zhang and J.-M. Jin, "Parallelized multilevel fast multipole algorithm for scattering by objects with anisotropic impedance surfaces," Int. J. Numer. Model., Electron. Netw., Devices Fields, vol. 28, no. 1, pp. 107119, Oct. 2015

[22] I. Hänninen, M. Pitkonen, K. Nikoskinen, and J. Sarvas, "Method of moments analysis of the backscattering properties of a corrugated trihedral corner reflector," IEEE Trans. Antennas Propag., vol. 54, no. 4, pp. 1167-1173, Apr. 2006.

[23] S. Rao, D. Wilton, and A. Glisson, "Electromagnetic scattering by surfaces of arbitrary shape," IEEE Trans. Antennas Propag., vol. 30, no. 3, pp. 409-418, May 1982.

[24] W. Gordon, "Far-field approximations to the Kirchoff-Helmholtz representations of scattered fields," IEEE Trans. Antennas Propag., vol. 23 , no. 4, pp. 590-592, Jul. 1975.

[25] — "High frequency approximations to the physical optics scattering integral," IEEE Trans. Antennas Propag., vol. 42, no. 3, pp. 427-432, mar 1994.

[26] J. M. Song and W. C. Chew, "Multilevel fast-multipole algorithm for solving combined field integral equations of electromagnetic scattering," Microwave and Opt. Technol. Letters, vol. 10, no. 1, pp. 14-19, Sep. 1995.

[27] T. Malas and L. Gürel, "Schur complement preconditioners for surface integral-equation formulations of dielectric problems solved with the multilevel fast multipole algorithm," SIAM J. Scientific Computing, vol. 33, no. 5, pp. 2440-2467, Jan. 2011.

[28] P. Ylä-Oijala, B. Kong, and S. Järvenpää, "Modeling of resonating closed impedance bodies with surface integral equation methods," IEEE Trans. Antennas Propag., vol. 67, no. 1, pp. 361-368, Jan. 2019.

[29] S. Yan and J.-M. Jin, "Self-dual integral equations for electromagnetic scattering from IBC objects," IEEE Trans. Antennas Propag., vol. 61, no. 11 , pp. 5533-5546, Nov. 2013.

[30] B. Kong, P. Ylä-Oijala, M. D. Astorino, H. Wallén, and A. Sihvola, "Analysis of scatterers with discontinuous impedance boundary condition using surface-integral-equation method," in 2018 12th International Congress on Artificial Materials for Novel Wave Phenomena (Metamaterials), Aug. 2018, pp. 225-227. 